\title{
ANALYSIS OF SHADING PATTERN OF SOLAR PANELS
}

\author{
Gargi Sarkar ${ }^{1}$, Kanchan Bakade ${ }^{2}$ \\ ${ }^{1}$ Student, Electronics and Telecommunication, NMIMS MPSTME, Maharashtra, INDIA \\ ${ }^{2}$ Assistant Professor, Electronics and Telecommunication, NMIMS MPSTME, Maharashtra, INDIA
}

\begin{abstract}
Abstract-The shading pattern of a Solar Panel if analyzed helps to improve the performance of a Solar panel. Shades on top of a Solar Panel are one of the major factors which causes disturbance on a solar panel. This paper helps analyze the shading on a solar panel which is caused by steady clouds. This may help to increase the efficiency of a solar panel. Various methods of Image processing technique is used to get the desired output. Those methods include techniques like median filtering and various morphological functions like usage of structuring element which gives the desired output. The problem of Boundary Estimation of a shading patter on a solar panel is addressed. Short-time shading and lightning is not at all useful for solar power plant. If the solar panel is shaded then proper measures have to be taken to keep the efficiency intact else there would be a serious loss in the amount of current generated with the help of a solar panel. Here the pixel values are measured and thus the exact numbers of dark and bright pixels are calculated and even the pixel values are reconfigurated to get the correct boundary estimation. The bypass diode is used to bypass the shaded area on the panel, which in turn helps to increase the efficiency of a solar panel by a significant amount. This method takes care of processing all kinds of shade on the solar panel from whatever source the shade is generated from.This concept can be taken further to analyse the various other parameters for effective operation.
\end{abstract}

Key Words: Cloud; Solar power plant; Partial shading; Image processing; Efficiency; Bypass diode

\section{INTRODUCTION}

The use of solar energy dates from antiquity. Between 1907 and 1913 the American engineer F. Shumman developed solar driven hydraulic pumps. With the increasing availability of low-cost oil and natural gas, interest in solar energy for power production is needed. Much amount of work was not done on solar energy from 1915-1950, but the interest was initiated by the American Association for the Advancement of Science at Washington DC in 1949 in the section of "future energy sources". At that time the future potentials as well as the economic problems for solar power was discussed. The wide spread discussion of solar energy was done only after the oil-embargo that occurred in $1973[5]$.

The performance of a PV array can be affected by various factors, such as temperature, solar isolation, solar angel of incidence, shading, inclination, angel of panel and array configuration. The power output of a solar panel depends on the solar intensity and angel of a PV array. A PV array can get shaded or partially shaded by various factors such as trees, clouds, buildings etc [2].Thus monitoring of an PV array needs to be done for maximum output of a solar panel.

This method can be used for the boundary estimation of anything which would require an exact estimation and pixel count. After this boundary estimation method is compiled with, the solar panel could be attached with bypass diode to get the good results of the amount of current produced. Some researchers used to use blocking diode on a solar panel to stop the current from getting into an infinite loop in the circuit itself, but the blocking diode itself does not help to safeguard the solar cells or provide any protection to reduce the output current according to the shading patterns on the solar panels.

Thus bypass diodes are used which are far more useful than a blocking diode and provides complete safety against any fluctuations which may cause the peak voltage to change in a particular situation.

The maximum current obtained from an solar panels or an array does not only matter only on the size (the number of panels on an array) but also how the configurations (connections of the panels) and the way the shading pattern is handled by a solar panel. Thus all the factors have to be taken care for the increase in the efficiency.

\section{STIMULATION PROCEDURE}

The reason for using MATLAB is that it is available in most academic, research, and industrial organizations and considered useful for several engineering disciplines. It provides several features that can be used to simulate highly 
complex systems, electronic and power electronic circuits and

systems and distributed generation power systems. To get the input image a simple camera is used with a good pixel count, so the clarity of the image remains intact.[1]

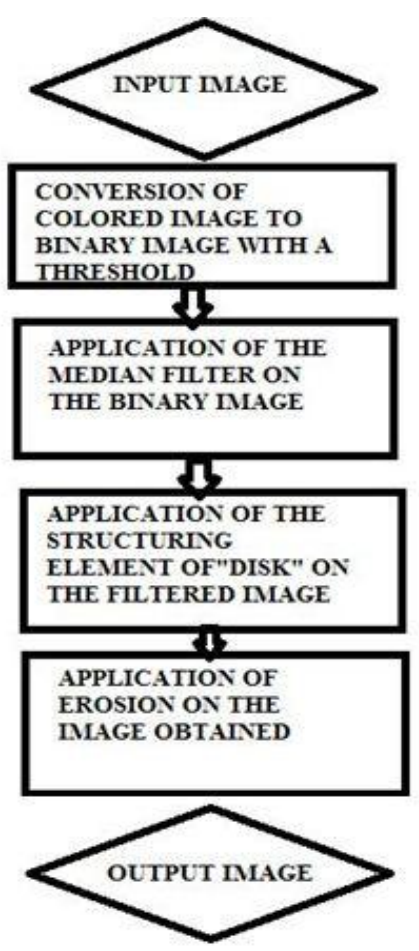

Figure 1- Flow Chart of the Stimulation Procedure

\subsection{Conversion of colored image to binary image}

Here a simple predefined function in MATLAB is used for the conversion of colored image to binary image.

\subsection{Application of median filter on the binary image}

This is a nonlinear digital filtering technique which is used to reduce and remove the noise (disturbances in an image).This stage usually aids to get better results in the following procedures to be performed. In this application method a window would be applied on the entire image. The pixel value in the entire image is taken into consideration and the median value of it is the intensity value of the output pixel.

\subsection{Application of structuring element}

This is a set of non linear functions usually related to shape or morphology of an image. The morphological functions do not deal with the intensity value of an image. Here the relative positions of the pixel values are dealt with according to the neighborhood pixels.
In this procedure another kind of window is applied all over the image. These windows are called structuring elements and these come in various shape. User uses whichever shape according to the usage.

For example, this code creates a flat, diamond-shaped structuring element.

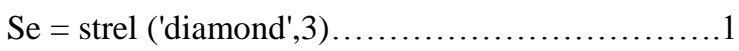

Decomposition: 3 STREL objects containing a total of 13 neighbours

Neighbourhood:-

$\begin{array}{lllllll}0 & 0 & 0 & 1 & 0 & 0 & 0 \\ 0 & 0 & 1 & 1 & 1 & 0 & 0 \\ 0 & 1 & 1 & 1 & 1 & 1 & 0 \\ 1 & 1 & 1 & 1 & 1 & 1 & 1 \\ 0 & 1 & 1 & 1 & 1 & 1 & 0 \\ 0 & 0 & 1 & 1 & 1 & 0 & 0 \\ 0 & 0 & 0 & 1 & 0 & 0 & 0\end{array}$

Comparisons of the structuring element along with the neighboring pixels are done and thus the approximate shape of the object in the image can be identified

The structuring elements work better in a binary image because the structuring element is usually a window of different shapes having intensity value of the image as either zero or one.

Here the structuring element of "disk" is being used here.

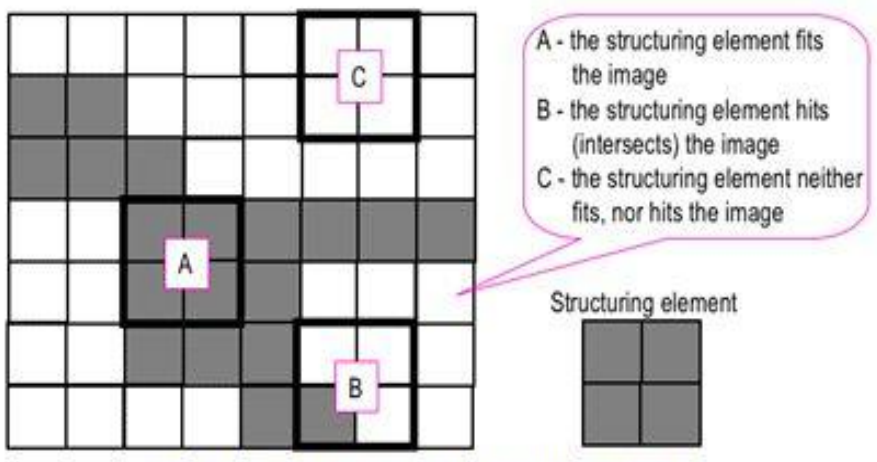

Probing of an image with a structuring element

(white and grey pixels have zero and non-zero values, respectively)

Figure 2- Structuring Element having the shape "square".

\subsection{Application of Erosion.}

Morphological operations include the application of erosion and dilation too.Erosion is used to remove pixels from the boundary of an image object,detected earlier in the procedure.

Erosion will occur according to the neighbourhood pixels of an image. Erosion might cause the boundary 
pixels of an image to either go dark or light according to the neighbourhood pixel intensity value[10]

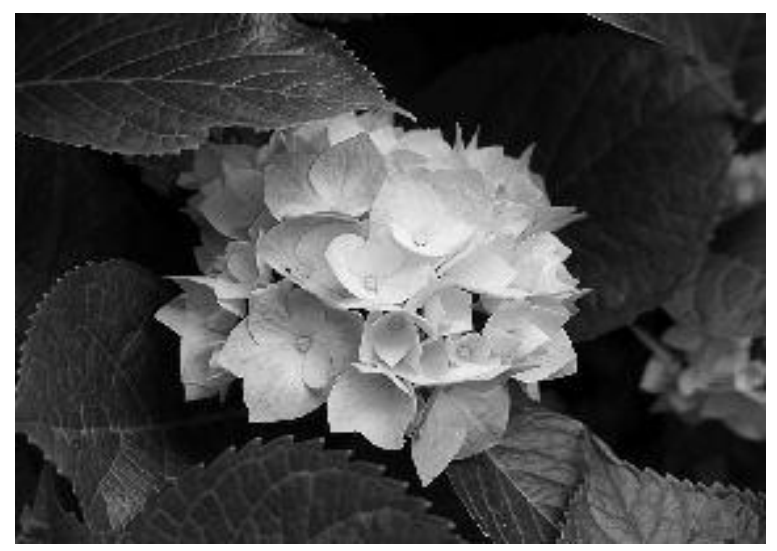

Figure 3-Original image of a flower

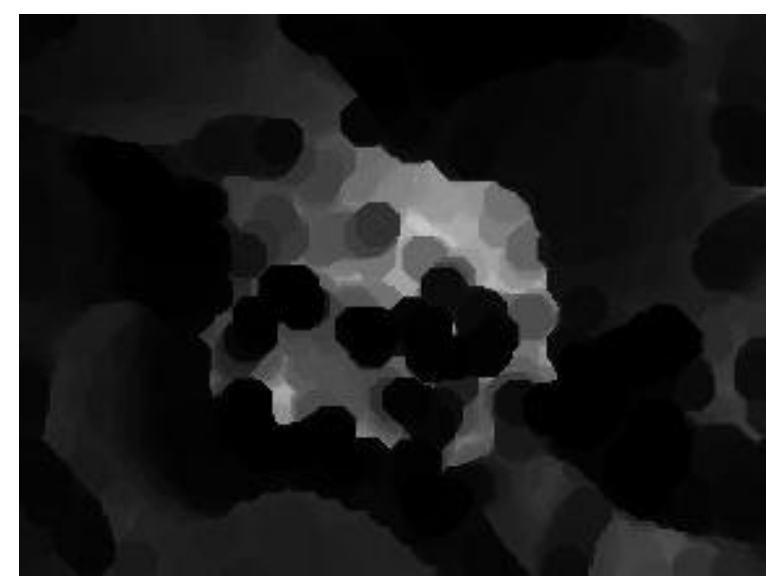

Figure 4-Eroded image of a flower

\subsection{OUTPUT IMAGE}

The ouput image obtained would be used to differentiate between the dark area and the light area and thus the bypass diode would work according to amount of light area available on the solar panel[3]

This way the solar cells which maybe shaded due to any obstruction may be easily byppassed with the help of bypass diode.

This analysis would help us to get the current with minimanl loss and thus a significant increase in efficiency.

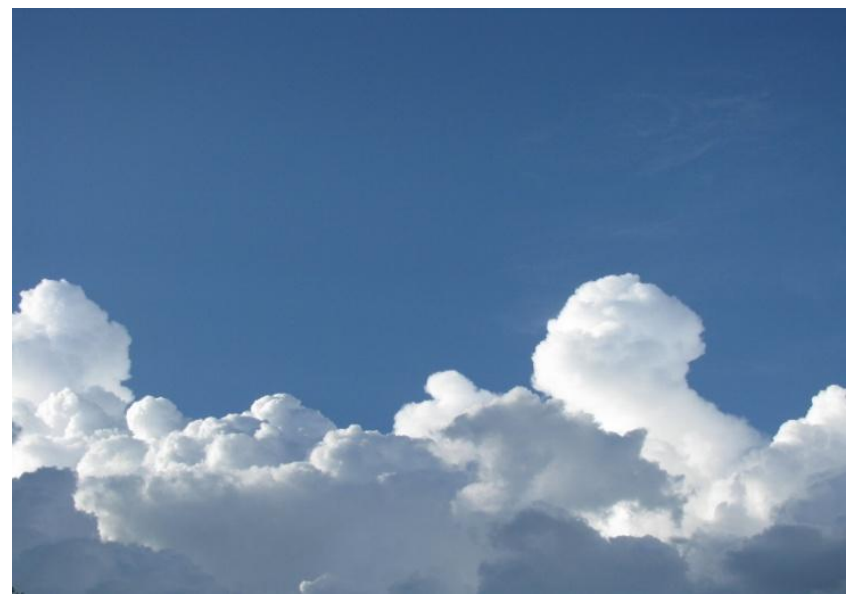

Figure 5-Original Picture of the Clouds

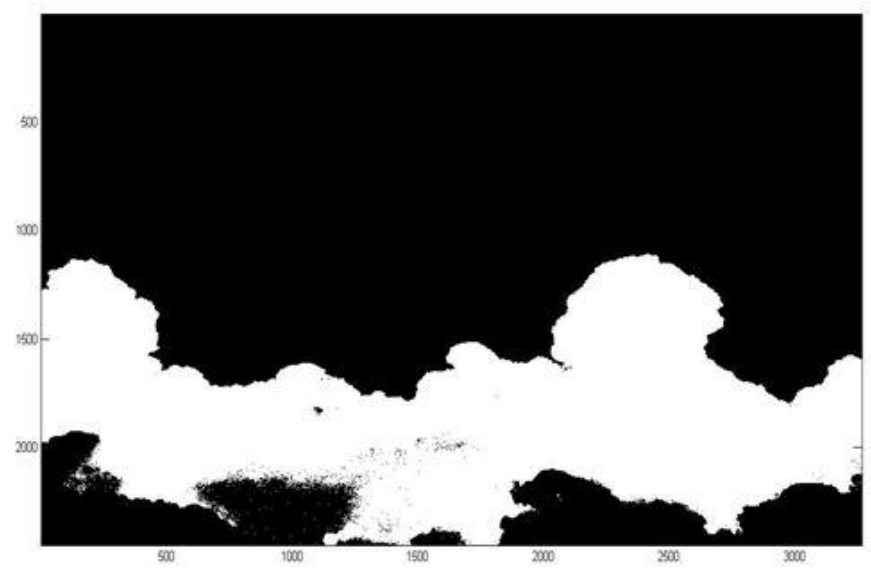

Figure 6-Boundary Estimation done by the algorithm used

\section{3 .SOLAR CELLS}

Photovoltaic is a method to generate electrical energy by converting solar energy into direct current electricity using semiconductors which exhibit photovoltaic effect.

Materials used for manufacturing of photovoltaic's are monocrystalline silicon, polycrystalline silicon, amorphous silicon, cadmium telluride, and copper indium gallium selenide/sulfide.

Photovoltaic is used for power generation by including multiple solar cells together and then forming an array of solar cells.[4]

The operation of a photovoltaic (PV) cell requires 3 basic attributes: 1.absorption of light, generating either electron hole pair or exitrons.2.separation of charge carriers of opposite types.3.The separate extraction of those carriers to an external circuit. 
Photovoltaic's is the direct conversion of light into electricity at the atomic level. Some materials exhibit a property known as the photoelectric effect that causes them to absorb photons of light and release electrons. When these free electrons are captured, an electric current result that can be used as electricity [12].

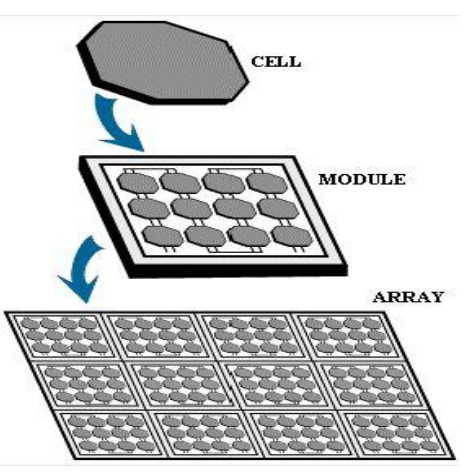

Figure 7-Forming of a PV array

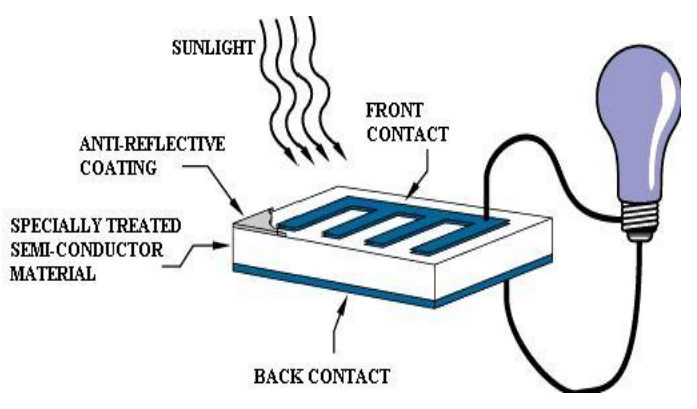

Figure 8-Working Of an PV array

\section{BYPASS DIODE}

Bypass diode are used on a solar panel for two reasons

i) To provide improved safety to the panels

ii) To help improve the performance and thus improve the efficiency of the Solar panel[11].

The primary reason that bypass diodes are used is to prevent the operating voltage of the module and the series string from getting too low if a panel has any partial shading. The shaded portion of the panel is, as the name suggests, bypassed [7].

A partially shaded SOLAR panel will always try to get the maximum output. Under shaded conditions also, a Solar Panel will always try and give maximum output, which causes the solar panel to give more output then possible, which causes loss of current and also increases the circuit temperature. Eventually causes the life of the circuit to reduce and also reduced outputs.

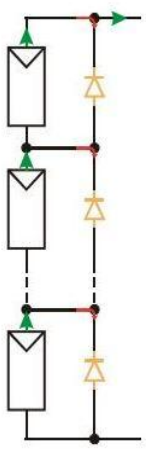

Normal Mode

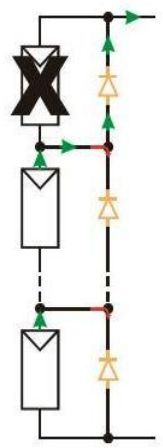

Bypass Mode (module partly shaded)

\section{Figure 9-Bypass diode in normal mode and in bypass mode}

So the bypass diode helps to reduce all the disadvantages of the solar panels and hence improve the overall output and thus improve efficiency.

The bypass diodes which are attached to the solar cell are usually at a reverse bias mode, thus allowing current to completely pass through it. The moment even a small percentage of the solar cell gets shaded due to any reason, the bypass diode switches from reverse bias to forward bias thus allowing the current to completely bypass the shaded cells.

This may cause the output voltage to reduce ,but atleast the effects of shaded cells causing damage to the whole array will significantly reduce[6-8]

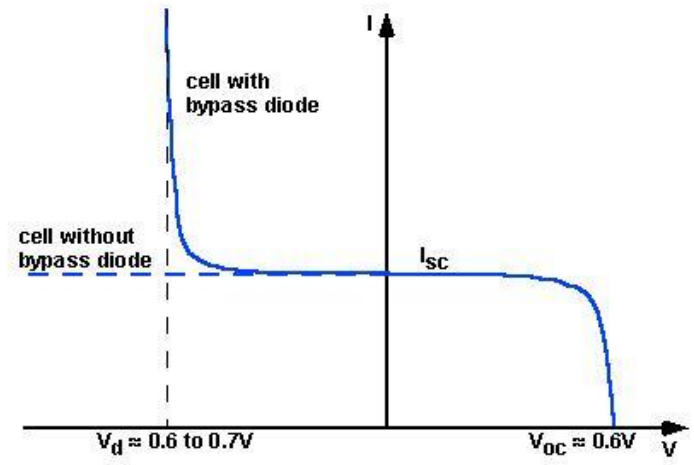

Figure 10-Output Characteristics of the solar panel with and without bypass diode. 
A single Bypass diode might be used for multiple solar cells together. That is usually done because a single bypass diode for every single cell would not be feasible because of the increased cost[9].

\section{TEST RESULTS}

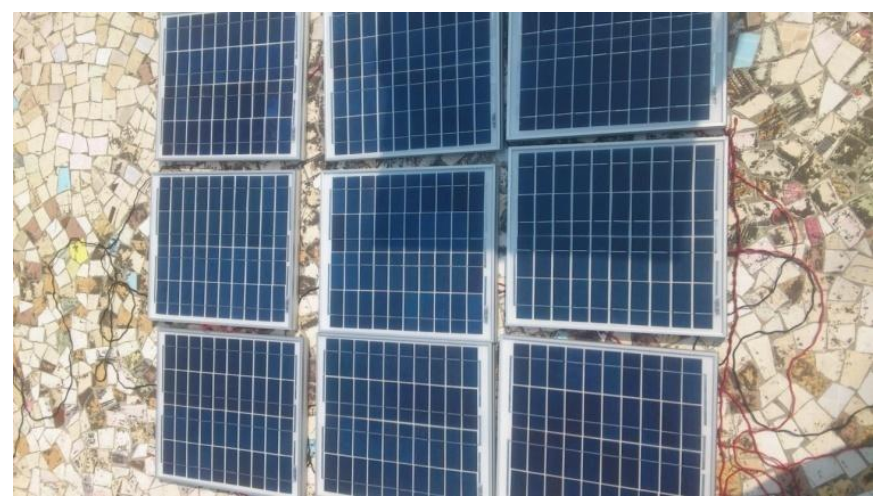

Figure 11- Original picture of the solar panel

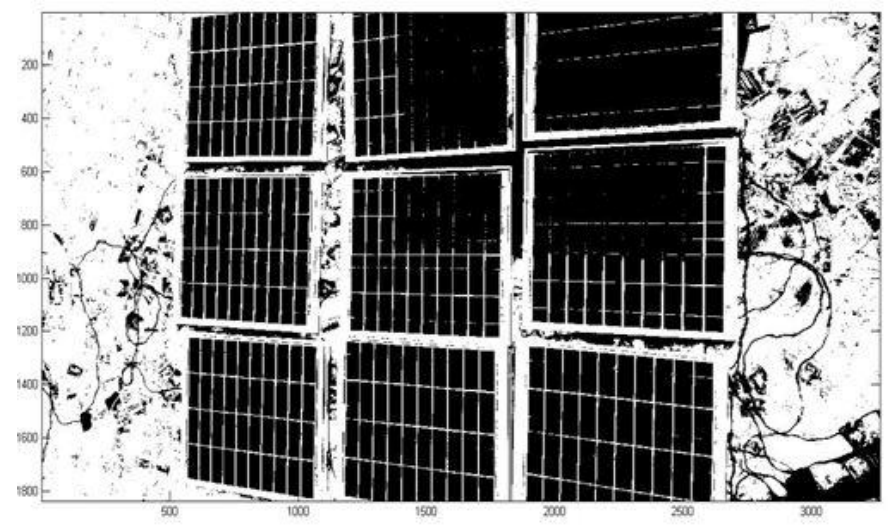

Figure 12-Boundary estimation done to the original picture of a solar panel.

\section{CONCLUSIONS}

This procedure consists of separating the dark pixels from the bright pixels and giving an exact count of the number of dark pixels and bright pixels. It is very important to understand the MATLAB program used to differentiate the dark and bright area. This procedure has a step wise routine which includes various methods to convert the binary image to complete black and white along with the pixel count.

After the boundary estimation is done, a bypass diode can be used to bypass the solar cells which are partially shaded and thus the efficiency of a solar panel can be improved

\section{ACKNOWLEDGEMENT}

Gargi Sarkar says thanks to her guide Kanchan Bakade and S.P.Duttagupta. A special thanks to Ashwini and Bhavani Patnaik because of their helpful insights and valuable advice.
Would like to say thanks to my classmates for their help and advice. A very special thanks to my friends and family for their unconditioned support and help.

\section{REFERENCES}

[1]. Hideaki Obane, Student Member, IEEE, Keiichi Okajima, Member, IEEE, Takashi Oozeki, Member, IEEE,and Takafumi Ishii "PV System With Reconnection to Improve Output Under Nonuniform Illumination" IEEE Journal (Volume:2 , Issuse 3),JULY 2012.

[2]. B.Patnaik, P.Sharma, E.Trimurthulu1, S.P.Duttagupta and V.Agarwal Department of Electrical Engineering, IITBombay, Mumbai, India"Reconfiguration Strategy For Optimization Of Solar Photovoltaic Array Under NonUniform Illumination Conditions"IEEE Journal,JUNE 2011.

[3]. Mario A. T. Figueiredo, Jose M. N. Leitao ,Instituto de Telecomunicaqdes and DEEC,Instituto Superior Tbcnico Lisboa Codex,Anil.k.Jain,department of computer science,Michigan University PORTUGAL "Adaptive $B$ Splines and Boundary Estimation" IEEE Computer Society conference,June 1997.

[4]. Lian Peng,Hua Fang, Ming Tang Department of Electronic Information and Control Engineering Guangxi University of Technology,Liuzhou, 545006, China "Solar power wireless monitoring system based on ARM7" Artificial Intelligence, Management Science and Electronic Commerce (AIMSEC), 2011 2nd International Conference on AUG 2011.

[5]. Ben-Gurion University of the Negev Faculty of Engineering Sciences Department of Nuclear Engineering."Real-Time Clouds Motion Analysis And Possibility Of Its Application To Solar Engineering" by Roman Palatnik.

[6]. Hiren Patel and Vivek Agarwal. MATLAB-Based Modeling to Study the Effects of Partial Shading on PV Array Characteristics. IEEE Transactions On Energy Conversion, VOL. 23, NO. 1, MARCH 2008.

[7].http://www.civicsolar.com/forum/9824/what-bypassdiode"Bypass Diode"

[8].http://solarprofessional.com/articles/designinstallation/q-a-bypass-diodes-improve-systemperformance-and-safety "Bypass Diode"

[9].http://pveducation.org/pvcdrom/modules/bypassdiodes“Working Of Bypass Diode”. 
[10] Desikachari Nadadur and Robert M. Haralick, Fellow,

IEEE- "Recursive Binary Dilation and Erosion Using

Digital Line Structuring Elements in Arbitrary

Orientations", IEEE TRANSACTIONS ON IMAGE

PROCESSING, VOL. 9, NO. 5, MAY 2000

[11] Atsushi Kajihara,Gunma Industrial Technology Center

Kamesatotyou 884-1 Maebashi, Tetsumi Harakawa

Maebashi Institute of Technology Kamisadorityou 460-

1Maebashi, "Model of Photovoltaic Cell Circuits under

Partial Shading”, 2005 IEEE TRANSACTIONS .

[12] http://science1.nasa.gov/science-news/science-at-

nasa/2002/solarcells ,"WORKING OF SOLAR CELLS",

\section{BIOGRAPHIES}

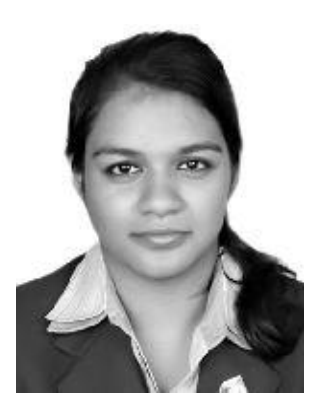

Gargi Sarkar completed her Btech in Electronics from Mukesh Patel School of Technology Management and Engineering, affiliated to NMIMS University in 2012.She has continued to study for her MTECH degree and is currently pursuing her master's from Mukesh Patel School of Technology and Management and would be graduating by 2014 .

Kanchan Bakade is currently working

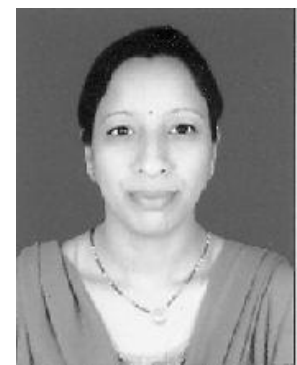
as an Assistant Professor in Mukesh Patel School of Technology Management and Engineering , affiliated to NMIMS University since from 2008. She has done her MTECH from IIT Roorkee in RF and Microwaves .Her area of interest are Wireless Communication ,Satellite Communication and RF. 